\title{
ON THE CHARACTERIZATION OF HILBERTIAN NORMS BY PROPERTIES OF HEIGHTS OF A TRIANGLE
}

BY

\section{IULIANA IONICĂ}

Abstract. In this paper we establish some characterizations of hilbertian norms in terms of heights vectors.

Mathematics Subject Classification 2000: 46B, 46C15.

Key words: smooth normed space, hilbertian norm, height of a triangle, Birchoff ortogonality.

\section{Introduction}

Let $(X,\|\cdot\|)$ be a real linear normed space and let us denote the directional derivatives of the norm by:

$$
n_{+}^{\prime}(x ; y)=\lim _{t \rightarrow \pm 0} \frac{\|x+t y\|^{2}-\|x\|^{2}}{2 t} \text {, for all } x, y \in X,
$$

limits which always exist ([3]). The basic properties of $n_{ \pm}^{\prime}$ to be frequently used are the following:

(i) $n_{ \pm}^{\prime}(x ; x)=\|x\|^{2}$ and $n_{ \pm}^{\prime}(x ; y) \leq\|x\| \cdot\|y\|, \forall x, y \in X$;

(ii) $n_{-}^{\prime}(x ; y) \leq n_{+}^{\prime}(x ; y), \forall x, y \in X$;

(iii) $n_{+}^{\prime}(-x ; y)=n_{+}^{\prime}(x ;-y)=-n_{-}^{\prime}(x ; y), \forall x, y \in X$;

(iv) $n_{+}^{\prime}(\alpha x ; \beta y)=n_{+}^{\prime}(\beta x ; \alpha y)=\alpha \beta n_{+}^{\prime}(x ; y), \forall x, y \in X$ and $\alpha, \beta \geq 0$;

(v) $n_{+}^{\prime}\left(x ; y_{1}+y_{2}\right) \leq n_{+}^{\prime}\left(x ; y_{1}\right)+n_{+}^{\prime}\left(x ; y_{2}\right), x, y_{1}, y_{2} \in X$; 
(vi) $n_{+}^{\prime}(x ; \alpha x+y)=n_{+}^{\prime}(x ; y)+\alpha\|x\|^{2}, \forall x, y \in X$ and $\alpha \geq 0$.

If $n_{-}^{\prime}(x ; y)=n_{+}^{\prime}(x ; y)$, for all $x, y \in X$, then we say that the norm is Gateaux differentiable, because $n_{+}^{\prime}(x, \cdot)$ is a linear continuous functional for each element $x \in X$. In this paper, whenever we will say that the norm is differentiable, we must understand the Gateaux differentiability.

In a linear normed space, if the norm is differentiable, then we say that the space is smooth. For instance, a linear normed space is smooth whenever his dual is strictly convex ([3], [9]).

In this paper we study some characterizations of linear normed spaces with the norm generated by an inner product using some metric properties of the heights of a triangle in a smooth linear normed space, properties which are well-known in the Euclidian geometry.

Most of the properties which use the directional derivatives lead us frequently to the symmetry of directional derivatives, which allow us to use the next well-known characterization:

Theorem 1.1. Let $(X,\|\cdot\|)$ be a real linear normed space, dim $X \geq 2$. Then $X$ is an inner product space if and only if for all linearly independent vectors $x, y$ in $X$

$$
n_{+}^{\prime}(x ; y)=n_{+}^{\prime}(y ; x) .
$$

According to (iv) it is sufficient that the property (2) to be satisfied only in the points $x, y \in X$ with $\|x\|=\|y\|=1$.

If the space is smooth, this property has been established for the first time by LEDUC in [11]. The non-smooth case was established by AMIR in [2]. In [12], PAPINI shows that the property (2) it is sufficiently to be satisfied in the next weaker expression, i.e. we have:

$$
\left|n_{+}^{\prime}(x ; y)\right|=\left|n_{+}^{\prime}(y ; x)\right|, \text { for all } x, y \in X \text { with }\|x\|=\|y\|=1
$$

(see also $[2,(2.5) \mathrm{pg} .18]$ ). In [14] we proved that the symmetry property (2) is sufficiently to be satisfied only in the pairs of points in which the norm is quite differentiable, taking into account that for an arbitrary norm, the set of differentiable points is dense in the space ([3], [7]). In fact, most of the characterizations of hilbertian norms in terms of directional derivatives established by different authors can be easily extended asking for those properties to be satisfied only in the points where the norm is quite differentiable (see, for instance [13]). 


\section{Some characterizations of hilbertian norms}

Let $(X,\|\cdot\|)$ be a general real linear normed space, endowed with the following orthogonality relation:

$$
x \perp y \Leftrightarrow\|x+t y\| \leq\|x\| \text {, for all } x, y \in X \text { and } t \in \mathbb{R},
$$

which usually it is known as Birkhoff orthogonality ([4]). The basic properties of this type of othogonality and the connections with other types of orthogonalities in a linear normed space were established, for instance, in [5], [6], [10].

Taking into account the property of minimum elements of a convex function (see, for example, [3], [7], [9]), if the space is smooth, this type of orthogonality can be characterized using the differential of the norm. Precisely :

$$
x \perp y \Leftrightarrow n^{\prime}(x, y)=0 \text {, for all } x, y \in X .
$$

In the case of the norms which are not necessarily Gateaux differentiable, the orthogonality condition can be characterized by directional derivatives of the norm:

$$
x \perp y \Leftrightarrow n_{-}^{\prime}(x ; y) \leq 0 \leq n_{+}^{\prime}(x ; y), \text { for all } x, y \in X .
$$

Let $(X,\|\cdot\|)$ be a real smooth linear normed space, $\operatorname{dim} X \geq 2, x, y \in X$ two linearly independent vectors and the triangle of vertices $0, x, y$.

Using the orthogonality properties, in [14] we defined the height vector by:

$$
h(x ; y)=y+\frac{n(y-x ; y)}{\|x-y\|^{2}}(x-y), \text { for all } x, y \in X .
$$

Indeed, the following orthogonality property holds: $(x-y) \perp h(x ; y)$, for all $x, y \in X$.

Some of the results presented in this paper are similarly to those obtained by GuiJarro and Tomas in [8], but they used another expression for the height vector, namely the one given in [1]:

$$
h(x ; y)=y+\frac{\|y\|^{2}-n_{+}^{\prime}(x ; y)}{\|x-y\|^{2}}(x-y), \text { for all } x, y \in X .
$$


In the case of smooth linear normed spaces it can be easily shown (see [14]) that the heights given by (6) and (7) are the same for any triangle if and only if the norm of the space is hilbertian. So, the results from this paper can not be derived by those obtained in [14] and reversely.

We will try to show in this paper that some characterizations established by GuiJarro and TOMAs in [8], are also conserved in the case of the height given by (6).

We know that in an inner product space, if we consider an isosceles triangle (meaning two sides with equal length), then the height vector $h(x ; y)$ divides the third side into two segments of equal length. We will see that this property characterizes the inner product spaces.

Lemma 2.1. Let $(X,\|\cdot\|)$ be a real smooth linear normed space, dim $X \geq 2$. Then $X$ is an inner product space if and only if for all linearly independent vectors $x, y$ in $X$ with $\|x\|=\|y\|$ we have:

$$
\left|n^{\prime}(y-x ; y)\right|=\left|n^{\prime}(x-y ; x)\right|
$$

Proof. If we assume that the property (8) holds then $n^{\prime}(y-x ; y)=$ $n^{\prime}(x-y ; x)$. Indeed, if it follows that $n^{\prime}(y-x ; y)=-n^{\prime}(x-y ; x)$, then $n^{\prime}(y-x ; y-x)=0$ and so we get $\|x-y\|^{2}=0$, meaning that $x=y$, which is impossible.

So, we obtain that $n^{\prime}(y-x ; y+x)=0$. Therefore by property $(5)$, we get $y-x \perp y+x$ and this is one of the characterizations of inner product spaces (see [2], pg.33, prop. 4.2.).

Theorem 2.1. Let $(X,\|\cdot\|)$ be a real smooth linear normed space, dim $X \geq 2$. Then the following conditions are equivalent:

(i) $X$ is an inner product space;

(ii) $\|h(x ; y)-y\|=\|h(y ; x)-x\|$, for all linearly independent $x, y \in X$ with $\|x\|=\|y\|$;

(iii) $\|x-y-h(x-y ;-y)\|=\|y-x-h(y-x ;-x)\|$, for all linearly independent $x, y \in X$ with $\|x\|=\|y\|$.

Proof. (i) $\rightarrow$ (ii) and (i) $\rightarrow$ (iii) are immediate. 
Firstly, if we assume (ii), using (6) we get:

$$
\left\|y+\frac{n^{\prime}(y-x ; y)}{\|x-y\|^{2}} \cdot(x-y)-y\right\|=\left\|x+\frac{n^{\prime}(x-y ; x)}{\|x-y\|^{2}} \cdot(y-x)-x\right\| .
$$

So, after a simple computation we obtain that $\left|n^{\prime}(y-x ; y)\right|=\left|n^{\prime}(x-y ; x)\right|$.

Using Lemma 2.1 it follows that $\mathrm{X}$ is an inner product space.

Let's assume now the condition (iii) and using (6) we get:

$$
\begin{aligned}
& \left\|x-y-\left(-y+\frac{n^{\prime}(-y-x+y ;-y)}{\|x-y+y\|^{2}} \cdot(x-y+y)\right)\right\| \\
& =\left\|y-x-\left(-x+\frac{n^{\prime}(-x-y+x ;-x)}{\|y-x+x\|^{2}}(y-x+x)\right)\right\|,
\end{aligned}
$$

which leads us to

$$
\left|\|x\|^{2}-n^{\prime}(x ; y)\right|=\left|\|y\|^{2}-n^{\prime}(y ; x)\right|, \forall x, y \in X \text { with }\|x\|=\|y\| .
$$

Using the property (i) of the norm's derivatives and taking into account that $\|x\|=\|y\|$, we get:

$$
n^{\prime}(x ; y) \leq\|x\|\|y\|=\|x\|^{2}, \quad n^{\prime}(y ; x) \leq\|y\|\|x\|=\|y\|^{2},
$$

which means that (11) is equivalent to:

$$
\|x\|^{2}-n^{\prime}(x ; y)=\|y\|^{2}-n^{\prime}(y ; x), \forall x, y \in X \text { with }\|x\|=\|y\| .
$$

Therefore we have $n^{\prime}(x ; y)=n^{\prime}(y ; x), \forall x, y \in X$ with $\|x\|=\|y\|$ and so $X$ is an inner product space, according to Leduc's characterization.

In the next two characterizations we will use some properties of angles connected to different heights of the triangles.

Theorem 2.2. Let $(X,\|\cdot\|)$ be a real smooth linear normed space, dim $X \geq 2$. Then $X$ is an inner product space if and only if for all linearly independent vectors $x, y$ in $X$ :

$$
\frac{\|x-h(x ; y)\|}{\|x\|}=\frac{\|x-y-h(x-y ;-y)\|}{\|x-y\|} .
$$

Proof. If $X$ is an inner product space, we can immediately verify that the condition (14) is satisfied. 
Now, if we assume (14), using (6) we get $h(x-y ;-y)=-y+\frac{n^{\prime}(x ; y)}{\|x\|^{2}} x$, for all $x, y \in X$. Replacing in (14), we obtain:

$$
\frac{\left\|x-y-\frac{n^{\prime}(y-x ; y)}{\|x-y\|^{2}}(x-y)\right\|}{\|x\|}=\frac{\left\|x-y+y-\frac{n^{\prime}(x ; y)}{\|x\|^{2}} \cdot x\right\|}{\|x-y\|} .
$$

After a simple computation we get:

$$
\left|\|x-y\|^{2}-n^{\prime}(y-x ; y)\right|=\left|\|x\|^{2}-n^{\prime}(x ; y)\right|, \forall x, y \in X,
$$

which is the same with:

$$
\left|n^{\prime}(x-y ; x)\right|=\left|n^{\prime}(x ; x-y)\right|, \quad \forall x, y \in X .
$$

Replacing $x-y$ by $u$ we obtain $\left|n^{\prime}(u ; x)\right|=\left|n^{\prime}(x ; u)\right|$, which means that $X$ is an inner product space according to property (3).

Remark 2.1. Let us specify that if $(X,\|\cdot\|)$ is a real smooth linear normed space with $\operatorname{dim} X \geq 2$, then the property (14) is equivalent with:

$$
\|x-h(y ; x)\|=\frac{\left|n^{\prime}(x ; x-y)\right|}{\|x-y\|},
$$

where $x, y$ are two linearly independent vectors in $X$.

Theorem 2.3. Let $(X,\|\cdot\|)$ be a real smooth linear normed space, dim $X \geq 2$. Then $X$ is an inner product space if and only if for all linearly independent vectors $x, y$ in $X$ with $\|x\|=\|y\|$ the following property is fulfilled:

$$
\frac{\|h(x ; y)\|^{2}}{\|y\|^{2}}+\frac{\|y-h(x ; y)\|^{2}}{\|y\|^{2}}=1 .
$$

Proof. If $X$ is an inner product space, we can immediately verify that the condition (17) is satisfied.

Now, if we assume (17), using (6) we obtain:

$$
\left\|y+\frac{n^{\prime}(y-x ; y)}{\|x-y\|^{2}}(x-y)\right\|^{2}+\left\|\frac{n^{\prime}(y-x ; y)}{\|x-y\|^{2}}(x-y)\right\|^{2}=\|y\|^{2}
$$

and after a simple computation we get:

(19) $\left\|n^{\prime}(y-x ; y) x-n^{\prime}(y-x ; x) y\right\|^{2}+n^{\prime}(y-x ; y)^{2}\|x-y\|^{2}=\|y\|^{2}\|x-y\|^{4}$. 
Interchanging $x$ with $y$ in (19) we also obtain:

(20) $\left\|n^{\prime}(x-y ; x) y-n^{\prime}(x-y ; y) x\right\|^{2}+n^{\prime}(x-y ; x)^{2}\|y-x\|^{2}=\|x\|^{2}\|y-x\|^{4}$.

Taking into account that we supposed from the beginning that $\|x\|=\|y\|$ and having the difference between (19) and (20), we get:

$$
\left[\left|n^{\prime}(x-y ; x)\right|^{2}-\left|n^{\prime}(y-x ; y)\right|^{2}\right]\|x-y\|^{2}=0 .
$$

As $x \neq y$, we obtain (8).

\section{REFERENCES}

1. Alsina, C.; Guijarro, P.; Tomás, M.S. - Some remarkable lines of triangles in real normed spaces and characterizations of inner product structures, Aequationes Math., 54 (1997), 234-241.

2. Amir, D. - Characterizations of Inner Product Spaces, Operator Theory: Advances and Applications, 20, Birkhäuser Verlag, Basel, 1986.

3. Barbu, V.; Precupanu, T. - Convexity and Optimization in Banach Spaces, D. Reidel Publ. Comp., Dordrecht, 1986.

4. Birkhoff, G. - Orthogonality in linear metric spaces, Duke Math. J., 1 (1935), 169-172.

5. DAy, M.M. - Some characterizations of inner-product spaces, Trans. Amer. Math. Soc., 62 (1947), 320-337.

6. DAy, M.M. - Normed Linear Spaces, Third edition, Ergebnisse der Mathematik und ihrer Grenzgebiete, Springer-Verlag, New York-Heidelberg, 1973.

7. Giles, J.R. - Convex Analysis with Application in the Differentiation of Convex Functions, Research Notes in Mathematics, 58, Pitman (Advanced Publishing Program), Boston, Mass.-London, 1982.

8. Guijarro, P.; Tomás, M.S. - Characterizations of inner product spaces by geometrical properties of the heights in a triangle, Arch. Math. (Basel), 73 (1999), 64-72.

9. Holmes, R.B.--Geometric Functional Analysis and its Applications, Graduate Texts in Mathematics, Springer-Verlag, New York-Heidelberg-Berlin, 1975.

10. James, R.C. - Orthogonality in normed linear spaces, Duke Math. J., 12 (1945), 291-302.

11. Leduc, M. - Caractérisation des espaces euclidiens, C.R. Acad. Sci. Paris Sér. A-B, 268 (1969), 943-946. 
12. PAPINI, P.L. - Inner products and norm derivatives, J. Math. Anal. Appl., 91 (1983), 592-598.

13. Precupanu, T. - Characterizations of Hilbertian norms, Boll. Un. Mat. Ital. B, 15 (1978), 161-169.

14. Precupanu, T.; IonicA, I. - Heights of a triangle in a linear normed space and Hilbertian norms, An. Ştiinţ. Univ. " Al.I. Cuza" Iaşi. Mat. (N.S.), 55 (2009), 35-47.

Received: 5.III.2009

Department of Mathematics,

University "Al.I. Cuza",

11, Bd. Carol I, 700506, Iaşi,

ROMANIA

ionica_iuliana@yahoo.com 